\title{
2007/56
}

Human capital, aggregation, and growth

Jakub Growiec 


\title{
CORE DISCUSSION PAPER
}

$2007 / 56$

\section{Human capital, aggregation, and growth}

\author{
Jakub GROWIEC ${ }^{1}$
}

August 2007

\begin{abstract}
The famous Mincer equation regressing log earnings on years of schooling is derived from a linear human capital accumulation equation at the individual level. Even if the cross-sectional Mincer equation holds at the level of individuals, it does not hold at the macro level of countries because aggregation of human capital has to take into account its vintage structure: human capital is embodied in people of different generations whose lifespan is finite. Finiteness of people's lives imposes also a limit on the potential of human capital accumulation to drive aggregate economic growth. Aggregate human capital accumulation may however become an engine of growth thanks to human capital externalities (knowledge spillovers). We use these findings to revisit the assumptions of the well-known Uzawa--Lucas growth model from an aggregation perspective.
\end{abstract}

Keywords: human capital accumulation, Mincer equation, aggregation, vintage structure, balanced growth

JEL classification: I20, J24, O40

${ }^{1}$ Warsaw School of Economics, Poland and CORE, Université catholique de Louvain, Belgium. E-mail: jakub.growiec@sgh.waw.pl

I acknowledge financial support from the Foundation for Polish Science. I am grateful to David de la Croix, Raouf Boucekkine, and Ingmar Schumacher for their helpful comments.

This paper presents research results of the Belgian Program on Interuniversity Poles of Attraction initiated by the Belgian State, Prime Minister's Office, Science Policy Programming. The scientific responsibility is assumed by the author. 


\section{Introduction}

Probably the most natural definition of human capital is "skills embodied in a worker" (Barro and Sala-i-Martin, 1995, p. 172). One key observation that lies at the foundation of human capital theory is that labor is not homogenous because people differ in their skills: an hour worked by a trained professional is worth much more than an hour worked by an amateur who performs the task for the first time. Human capital is accumulated, i.e. productivity of an hour worked by a given individual is increased, by formal schooling, training, and on-the-job learning.

Barro and Sala-i-Martin (1995, Chapter 5, p. 172) shed more light on the process of human capital accumulation by comparing it to technological progress:

We should emphasize, however, some respects in which the accumulation of human capital differs from the creation of knowledge in the form of technological progress. If we think of human capital as the skills embodied in a worker, then the use of these skills in one activity precludes their use in another activity: hence, human capital is a rival good. Since people have property rights in their own skills, as well as in their raw labor, human capital is also an excludable good. In contrast, ideas or knowledge may be nonrival - in that they can be spread freely over activities of arbitrary scale - and may in some circumstances be nonexcludable.

It follows that human capital is (i) embodied in people, (ii) rival, (iii) excludable, and therefore markedly different from ideas, knowledge, or a general "technology level".

Yet, not all consequences of this characterization of human capital are always recognized. Quite often it is forgotten that since human capital is embodied in people, it is lost upon death. Moreover, one should not expect newborn babies to inherit the human capital of their parents automatically. They have to learn the skills themselves, parents and teachers can only offer guidance and help.

Thus, when thinking about aggregate human capital accumulation, we have to go beyond the observation that it is accumulated through schooling, training, and on-the-job learning. We have also to account for the finiteness of life of individuals that embody human capital. Since people die and are born at different moments in time, aggregation requires us to account for the explicit vintage structure of human capital (cf. Boucekkine, de la Croix, and Licandro, 2002): heterogeneity in ages generates heterogeneity in human capital levels.

Section 2 of the current paper goes through the aggregation procedure in the simplest, and thus most transparent, case. To keep things as simple as possible, 
we ignore intra-cohort heterogeneity so that the only source of heterogeneity is the age of individuals, or equivalently, the cumulative amount of education they have received in their lives.

We argue that if one wants to obtain a log-linear (Mincerian) cross-sectional relationship between human capital and years of schooling, a linear human capital accumulation equation at the individual level should be assumed. However, even if the Mincerian relationship holds at the individual level, it is inevitably lost upon aggregation. This finding could explain why in empirical research, the Mincerian specification works much better at the micro level (see e.g. Mincer, 1974; Psacharopoulos, 1994, or Heckman, Lochner and Todd, 2003) ${ }^{1}$ than at the macro level of countries (e.g. Heckman and Klenow, 1997; Krueger and Lindahl, 2001; Bloom, Canning, and Sevilla, 2001). We also find that in our simplest case, aggregate human capital is constant over time unless the age structure of the society shifts or the unit efficiency of learning changes.

The second question asked here is whether human capital accumulation could potentially be conducive to long-run growth even though all people eventually die. This question, motivated by the aggregation perspective we are taking here as well as by the embodied character of human capital, is addressed in Section 3. We provide a list of possible reasons for human capital accumulation to drive, or at least facilitate, aggregate economic growth despite the fact that individuals are finitely-lived and all investment in embodied skills will eventually be lost. We conclude that human capital externalities head this list but are nevertheless not sufficient for balanced growth.

At this point, we turn to a critical re-assessment of the famous Uzawa-Lucas model (Uzawa, 1965; Lucas, 1988) of growth driven by human capital accumulation by infinitely-lived individuals (or alternatively, by accumulation of disembodied human capital). In its simplest specification, the model fails to capture the depreciation of aggregate human capital due to births and deaths of people embodying human capital. However, it can be reconciled with aggregation if knowledge spillovers are strong enough: there exists then an asymptotic balanced growth path around which aggregate human capital accumulation might be approximated by the linear differential equation posed in the Uzawa-Lucas model.

Sections 2 and 3 provide the basic argument in this paper. First, we show under which circumstances the Mincer equation is supposed to hold at the micro level. Second, we show why it is never supposed to hold at the macro level of countries after the vintage structure of human capital has been properly accounted for. Finally, we find that human capital accumulation could enter the

\footnotetext{
${ }^{1}$ Heckman, Lochner and Todd (2003) begin the abstract of their paper with the sentence: "The Mincer earnings function is the cornerstone of a large literature in empirical economics."
} 
engine of aggregate economic growth only under certain specific conditions. As we discuss in the following Section 4, one of such conditions is the existence of knowledge spillovers in human capital accumulation. In Section 5 we apply our results to revisit the assumptions of the Uzawa-Lucas model. Section 6 provides several qualifications and robustness checks for the main argument. More precisely, we allow for non-linearities at the individual level, more realistic survival laws, and more sophisticated time profiles of learning and working in the individuals' life-cycles. Section 7 concludes.

\section{The basic argument}

In the first step of our basic argument, we shall assume a linear differential equation of human capital accumulation at the individual level, and derive from it a cross-sectional relationship between human capital and schooling effort that follows closely Mincer $(1958,1974)$. Our knife-edge linearity assumption imposes the restriction that the increments to one's skills are exactly proportional to the skills one has already mastered. The coefficient of proportionality is then interpreted as unit efficiency of schooling (or on-the-job learning), multiplied by the current learning (working, respectively) effort made by the individual.

We shall see shortly that if the increments to one's skills are proportional to the stock of her previously acquired skills, and if earnings are proportional to skills, then the Mincer earnings equation should work. Of course, there is a long list of reasons why Mincerian regressions may fail, for example: (i) the increments to one's skills may be more or less than proportional to the previously acquired skills (cf. Psacharopoulos, 1994; Belzil and Hansen, 2002), (ii) earnings may not reflect individuals' human capital properly, (iii) raw years of schooling may hide the differences in learning effort across individuals and time, (iv) different schools and teachers may offer different efficiencies in teaching, (v) there might arise educational mismatches where the skills acquired at school are not useful for the current job (Psacharopoulos, 1994), (vii) the average efficiency of schooling may shift over time, an effect that is not captured when taking cross-sections over people of different generations (cf. Arias and McMahon, 2001; Heckman, Lochner, and Todd, 2003). Nevertheless, if neither of these points is a serious problem, the Mincerian specification should work.

Fairly surprisingly, under the same set of assumptions, the Mincerian specification should not work in macro-level cross-country regressions. The reason for this failure is that the data on average human capital levels are built using an aggregation procedure which implicitly takes into account the age structure of the society. Often overlooked when specifying cross-country regressions is the fact that experienced and educated people die and are replaced by next generations 


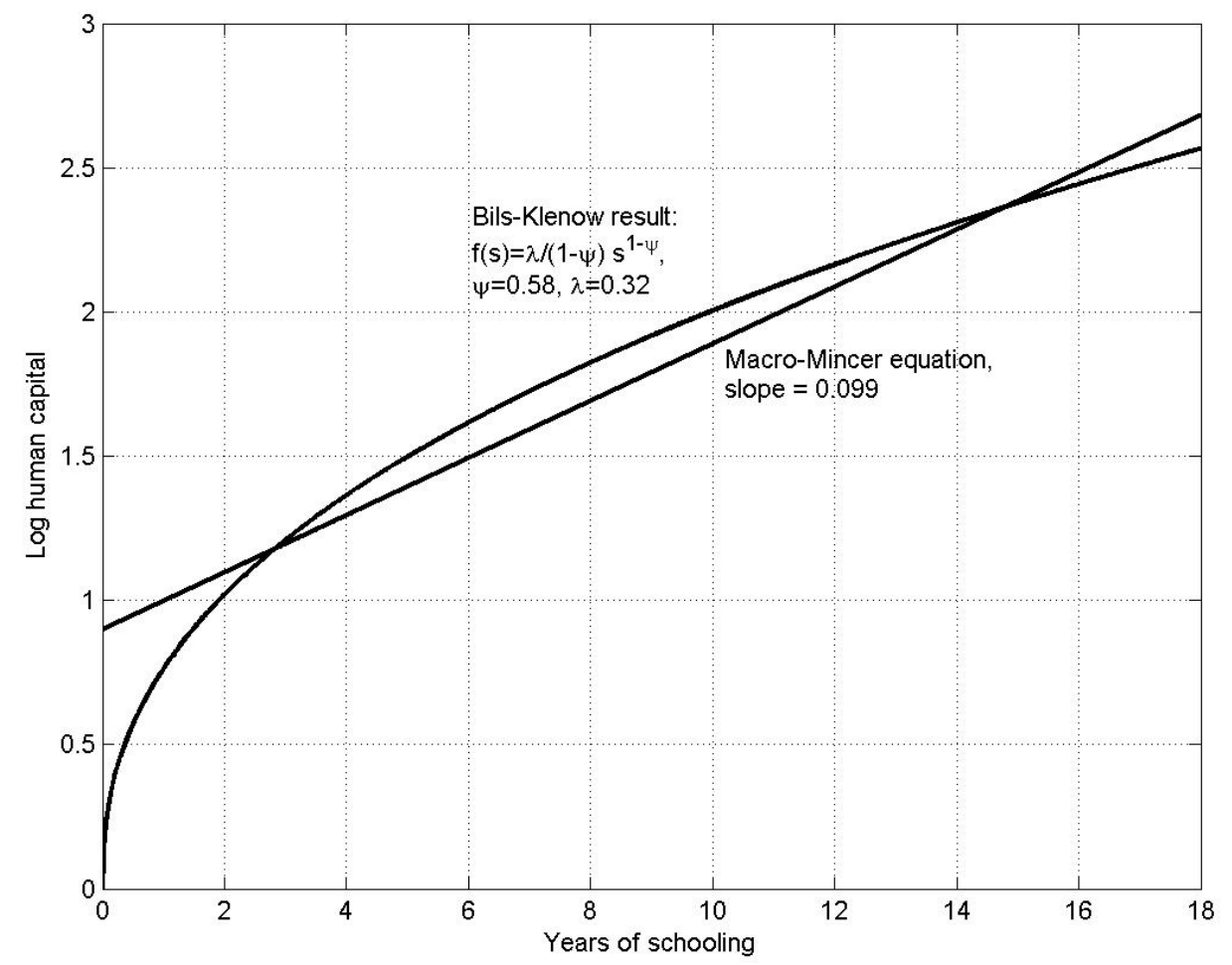

Figure 1: The macro Mincer equation might not hold even if the micro Mincer equation holds. Bils and Klenow's (2000) concavity result plotted against the log-linear benchmark.

that have to learn first the skills their ancestors already had. Thus, in aggregate terms, a substantial part of the investment in schooling is in fact replacement investment. The average level of human capital is increased by schooling, training, and on-the-job learning, but at the same time it is decreased by deaths of the human-capital-rich and births of the human-capital-poor. It is not possible to preserve the log-linear relationship between earnings and years of schooling upon aggregation (see Figure 1). This result stands in sharp contrast to a number of papers which ignore the dependence of this aggregate relationship on the age structure of the society and postulate the macro Mincer equation directly: notably, Heckman and Klenow (1997), Bloom, Canning, and Sevilla (2001), and Krueger and Lindahl (2001). ${ }^{2}$ Instead of estimating aggregate Mincer equations,

\footnotetext{
${ }^{2}$ To be fair, the aggregate Mincer equation is not uniformly accepted in the literature. A number of works question it on diverse bases - e.g. Hall and Jones (1999) and Bils and Klenow (2000) emphasize the macro-level finding of log earnings being concave in years of schooling.
} 
one could try to derive specifications which are consistent with the underlying aggregation procedure and thus take into account the age structure of the considered society. Another issue arising here which is not handled in the current paper is intra-cohort heterogeneity in human capital levels, a phenomenon which could also potentially influence the shape of the aggregative earnings-schooling relationship. One could also use more general functional forms which could help identify the true macro-level relationship between average years of schooling and average earnings (cf. Hall and Jones, 1999; Bils and Klenow, 2000).

The announced result of incompatibility of aggregate Mincer equations with intergenerational aggregation stands in opposition also to the popular practice of carrying the cross-sectional Mincerian relationship forward to dynamic growth models with a representative agent (cf. Jones, 2005). The static equation $h=e^{\psi \ell_{h}}$ where $h$ is the human capital level of the agent and $\ell_{h}$ is the time share of schooling, cannot be reconciled with plausible demographics (which imply the need to aggregate age-specific human capital over all generations).

Another finding is that in a stationary environment, where the age structure of the society is stable over time (even though the population itself may be growing or declining) and where there is no technological progress, we should observe a constant average human capital level. Since all people eventually die, human capital accumulation cannot work as an engine of aggregate growth despite the strictly linear differential equation at the individual level.

\subsection{Deriving the micro-level Mincer equation}

Assume that human capital embodied in each person evolves according to a linear differential equation - exactly the one which Lucas (1988) used in his aggregate specification. At each instant of time, the individual's human capital $h$ is increased by the quantity $\dot{h}=\left(\lambda \ell_{h}+\mu \ell_{Y}\right) h$. The first component of the increment relates to the education effort made by the individual $(\lambda>0$ denotes the constant unit efficiency of education), while the second component relates to work effort: hours worked increase work experience and thus labor productivity $(\mu \geq 0$

\footnotetext{
Above all, there exist reasons different from aggregation which affect the relationship between aggregate human capital and average years of schooling, such as measurement error (de la Fuente and Doménech, 2006), unexplained heterogeneity in rates of return across countries (Psacharopoulos, 1994), heterogeneity in rates of return across levels of education (Hall and Jones, 1999), reverse causality (Bils and Klenow, 2000), and the complementarity between human capital and technology. In many studies, a log-log relationship (a Cobb-Douglas production function) between years of schooling and GDP is assumed instead of a log-linear one (see e.g. Mankiw, Romer, and Weil, 1992; Benhabib and Spiegel, 1994; de la Fuente and Doménech, 2006). Human capital is then simply identified with years of schooling.
} 
describes the pace at which experience is acquired). ${ }^{3}$ The individual is endowed with a fixed flow of time to divide between learning, working, and other activities (such as leisure or child rearing). We normalize it to unity and consequently impose the restriction that $\ell_{h}, \ell_{Y} \in[0,1]$ as well as $\ell_{h}+\ell_{Y} \leq 1$.

Using more precise notation, we write that each individual born at time $t$, being currently at the age of $\tau$, accumulates her human capital according to

$$
\frac{d}{d \tau} h(t, \tau)=\left(\lambda \ell_{h}(\tau)+\mu \ell_{Y}(\tau)\right) h(t, \tau)
$$

Let us further assume that people are born with a fixed initial level of human capital $h(t, 0)=h_{0}>0$ that does not depend on time $t$ at which the individual is born. The constancy of $h_{0}$ follows directly from its interpretation: $h_{0}$ is the natural level of useful abilities and skills, prior to all development. This is a rather innocuous, yet important assumption, since if for any reason (i.e. better nutrition, natural selection, genetic engineering, etc.), $h(t, 0)$ could grow over time, some of our results will be overturned (see the discussion in Section 3).

The differential equation (1) can be solved for $h(t, \tau)$ yielding

$$
h(t, \tau)=h_{0} \exp \left(\lambda \int_{0}^{\tau} \ell_{h}(s) d s+\mu \int_{0}^{\tau} \ell_{Y}(s) d s\right) .
$$

Equation (2) is a variant of the Mincerian specification: it says that log human capital depends linearly both on total schooling effort and on cumulative work experience.

As a corollary, we note the following. Assuming that an individual of age $\tau$ first engages in full-time education $\left(\ell_{h}=1\right)$ for time $\tau_{h}$ and then works full-time $\left(\ell_{Y}=1\right)$ for time $\tau_{Y}$, with $\tau_{h}+\tau_{Y} \leq \tau$, we obtain a particularly nice result:

$$
h=h_{0} \exp \left(\lambda \tau_{h}+\mu \tau_{Y}\right) \quad \Leftrightarrow \quad \ln h=\ln h_{0}+\lambda \tau_{h}+\mu \tau_{Y} .
$$

Under the assumption that wages are proportional to human capital, (3) is directly the Mincer wage equation (Mincer, 1974): ${ }^{4} \log$ wages regressed on years of

\footnotetext{
${ }^{3}$ A similar derivation has been put forward by Mincer (1974) and later discussed by Heckman, Lochner, and Todd (2003). The difference is that our version of the Mincer's discrete-time equation $h_{t+1}=\left(1+\rho_{t}\right) h_{t}$ (which adds also the on-the-job training part) is presented here as a restrictive assumption on schooling technology, while in those two works it is presented as an accounting identity. However, these authors immediately assume that $\rho_{t}-$ the rate of return on formal schooling - is constant over all years of schooling. Even if we indeed think of the individual's human capital accumulation equation as of an accounting identity, then this constancy is the key restriction. It does not follow from the identity but is imposed arbitrarily.

${ }^{4}$ The difference is that we omit the square term in work experience here. This is because for simplicity, we have abstracted from the finding that on-the-job training is characterized not by constant but by decreasing returns. See equation (1) in Krueger and Lindahl (2001).
} 
schooling and years of work experience. This is a micro-level equation because it refers to wages, years of schooling, and years of work experience as measured for a given individual at a single moment in time. It should be noted that equation (2) applies only to individuals born at the same time $t$. It holds also for a crosssection of people born at different times only if the efficiency parameters $\lambda$ and $\mu$ are constant over time (see Arias and McMahon, 2001).

Since each individual's human capital depends on her age but not on the time at which she was born, that is $h(t, \tau)$ does not depend on $t$, it follows automatically that under a stable age structure, the average level of human capital in the society $\bar{h}(t)$ will not depend on $t$ either. Hence, it will be constant over time. This result should be contrasted with the human capital-based macro growth literature that assumes aggregate human capital to grow over time, e.g. Uzawa (1965), Lucas (1988, 1993), Barro and Sala-i-Martin (1995, Chapter 5), Gong, Greiner and Semmler (2004), and numerous other articles in this vein. In fact, $\bar{h}(t)$ is constant over time here despite the fact that there is balanced growth in personal human capital as each individual ages.

\subsection{Aggregation across individuals}

Let us now turn to demographics. To keep the basic argument as simple as possible, assume that at each moment in time, spanning from minus to plus infinity, $t \in(-\infty,+\infty)$, there exists a continuum of people of measure $N(t)$. Suppose for simplicity that the birth rate is constant, age-invariant, and equal to $b>0$ - that is, the number of births at each moment in time $t$ is proportional to total population and equal to $b N(t)$. We shall also tentatively limit ourselves to the simplest "perpetual youth" case implying that the hazard rate of death faced by each individual is constant, independent of age, and equal to $d>0$ (cf. Blanchard, 1985). This means that the unconditional probability of surviving to an age of $\tau$ and the conditional probability of surviving an additional $\tau$ years are equal and decreasing exponentially: $m(\tau)=e^{-d \tau} \cdot{ }^{5}$ Given these simplifying assumptions and the Law of Large Numbers, it follows that the population growth rate is deterministic, constant, and equal to $\hat{N}(t)=b-d$ for all $t$. If $N(0)=N_{0}$ then there are $N(t)=N_{0} e^{(b-d) t}$ people alive at $t$.

The age structure of the population alive at $t$ is easy to compute. Naturally, to be $\tau$ years old at $t$, one has to (i) be born at $t-\tau$, and (ii) survive to the age of $\tau$. This implies that there are $P(t, \tau)$ people aged $\tau$ in the population, with

$$
P(t, \tau)=b N(t-\tau) m(\tau)=b N_{0} e^{(b-d)(t-\tau)} e^{-d \tau}=N(t) b e^{-b \tau} .
$$

\footnotetext{
${ }^{5}$ Using more realistic survival laws, implying that youth is "fleeting" rather than "perpetual", i.e. Boucekkine, de la Croix, and Licandro (2002) or Faruqee (2003), does not contradict the main message conveyed herein. See Section 6 for a more detailed discussion of this issue.
} 
The average level of human capital in the society is given by

$$
\bar{h}(t)=\int_{0}^{\infty} \frac{P(t, \tau) h(t-\tau, \tau)}{N(t)} d \tau=b \int_{0}^{\infty} h(t-\tau, \tau) e^{-b \tau} d \tau .
$$

To shed more light on the above result, let us provide a simple example that offers an explicit solution. ${ }^{6}$ Namely, if individuals always spend constant percentages of their time endowment on learning and working, $\bar{\ell}_{h}$ and $\bar{\ell}_{Y}$ respectively, we get:

$$
\bar{h}(t)=b \int_{0}^{\infty} h_{0} e^{\left(\lambda \bar{\ell}_{h}+\mu \bar{\ell}_{Y}\right) \tau} e^{-b \tau} d \tau=\frac{b h_{0}}{b-\lambda \bar{\ell}_{h}-\mu \bar{\ell}_{Y}}
$$

under the assumption that $b>\lambda \bar{\ell}_{h}+\mu \bar{\ell}_{Y}$ so that the few arbitrarily old people with arbitrarily high human capital levels (existence of such individuals is an unrealistic implication of the "perpetual youth" survival law that does not impose any upper bound on people's lifespans) do not dominate the population, causing the average human capital level to diverge. ${ }^{7}$

Two things have to be noted in relation to this example. First, we obtain usual comparative statics: $\bar{h}(t)$ increases with $\lambda, \mu, \bar{\ell}_{h}, \bar{\ell}_{Y}, h_{0}$ and decreases with $b$. Second, the derived hyperbolic pattern of dependence on the efficiency parameters (with $\bar{h}(t) \rightarrow \infty$ as $\lambda \bar{\ell}_{h}+\mu \bar{\ell}_{Y} \rightarrow b$ ) is not a robust finding but rather an artifact of the assumed "perpetual youth" survival law. Nevertheless, aggregate human capital will typically not follow the exponential (Mincerian) pattern of dependence on years of schooling and work experience even if such pattern is found in individual data.

Before we relax the "perpetual youth" assumption and begin the discussion on the circumstances under which the macro-level Mincerian equation could potentially hold, we have to remark on the consequences of assuming a stationary age structure.

\footnotetext{
${ }^{6}$ Implicit in our aggregation exercise is the assumption that skill levels are perfectly substitutable. We simply calculate the arithmetic mean of individual human capital levels. However, workers with different skill levels might not be perfectly substitutable in production. Pandey (2005) argues that the actual elasticity of substitution between skilled and unskilled labor is not infinite, but around 4 when estimated using a worldwide cross-country sample. It drops to $\approx 2.22$ when only low-income countries are considered, and rises to around 50 if only highincome countries are considered. Furthermore, Pandey (2005) indicates that "[t]he results in the literature are extremely sensitive to the specification of the aggregate production function. In particular, (...) the elasticity of substitution between skilled and unskilled labor used to aggregate the two types of labor plays a crucial role." (p. 1) We leave this problem for further work.

${ }^{7}$ Manuelli and Seshadri (2005) carry out a similar aggregation exercise for their human capital-based general equilibrium model.
} 


\subsection{Stationary age structure}

Whatever survival law $m(\tau)$ we choose, a stationary age structure implies that the death rate is constant and equal to $d^{*}$ where $d^{*}$ solves the implicit equation

$$
d+\int_{0}^{\infty} b e^{(b-d) \tau} m^{\prime}(\tau) d \tau=0
$$

It should be noted that for any survival law, equation (7) is automatically satisfied if $b=d$, i.e. if population is constant over time. ${ }^{8}$

However, the implicit equation (7) typically has two solutions. To characterize these solutions more precisely, we denote the life expectancy at birth as

$$
E=-\int_{0}^{\infty} \tau m^{\prime}(\tau) d \tau .
$$

Using this notation, we obtain the following result. If $b E>1$ then the nontrivial solution of $(7)$ implies $b>d$, indicating a growing population. Conversely, if $b E<1$ then $b<d$ holds, indicating a declining population. In the special case $b E=1$, the zero growth solution $b=d$ is unique. ${ }^{9}$

This result is very intuitive: it means that population can grow steadily, preserving the shape of the age distribution, if and only if the average number of offspring per person is greater than one. Conversely, if the average number of offspring per person is less than one, the population can steadily decline. Obviously, the average number of offspring per person is directly $b E$ here - the instantaneous fertility rate times the life expectancy.

We are now equipped with the tools necessary to announce the incompatibility of the macro Mincer equation (Heckman and Klenow, 1997; Krueger and Lindahl, 2001) with aggregation.

\subsection{The incompatibility of the macro Mincer equation with aggregation}

Let us now ask the converse question. If in the "perpetual youth" case, the macro-level Mincer equation fails but a hyperbolic function is derived instead, then what should be the survival function $m(\tau)$ to assure that the macro Mincer equation holds?

\footnotetext{
${ }^{8}$ Since $m$ is a survival law, i.e. $1-m$ is a cumulative distribution function concentrated on $[0,+\infty)$, we always have $\int_{0}^{\infty} m^{\prime}(\tau) d \tau=-1$.

${ }^{9}$ The proof of this result uses the fact that the left-hand side of (7) is continuous and concave in $d$ and that it is equal to zero for $b=d$.
} 
We find that under a stationary age structure, it is not possible unless the survival function depends on years of schooling in one crucial and arguably implausible way.

Assume again that the individuals spend constant percentages of their time endowments on learning and working, $\bar{\ell}_{h}$ and $\bar{\ell}_{Y}$ respectively; use the notation $\Phi=\lambda \bar{\ell}_{h}+\mu \bar{\ell}_{Y}$ for further simplification. In mathematical terms, our current question reads: for what function $m(\tau)$, it holds that

$$
\bar{h}(t)=\xi e^{\Phi}, \quad \text { with } \xi>0 ?
$$

The answer is straightforward. Since we pose

$$
\bar{h}(t)=b h_{0} \int_{0}^{\infty} \frac{N(t-\tau)}{N(t)} m(\tau) e^{\Phi \tau} d \tau=b h_{0} \int_{0}^{\infty} m(\tau) e^{(\Phi-b+d) \tau} d \tau=\xi e^{\Phi},
$$

it follows that the sought $m(\tau)$ function must satisfy

$$
m(\tau)=\frac{\xi}{b h_{0}} e^{(b-d) \tau} e^{(1-\tau) \Phi} f(\tau),
$$

where $f(\tau)$ is a probability density function (pdf) concentrated on $[0,+\infty)$. In principle, it can be just any pdf; the only restrictions are that $m(0)=1, m^{\prime}(\tau)<$ 0 , and that $f(\tau)$ cannot depend on $\Phi .^{10}$

In order for the macro-level Mincer equation to hold, $m(\tau)$ must depend on $\Phi$ in one crucial way: uniquely through the factor $e^{(1-\tau) \Phi}$. This is not only a very stringent condition but also an arguably implausible one, because it implies that for ages $\tau<1$, survival probabilities increase with learning effort, while for ages $\tau>1$, they decrease with learning effort. In reality, though, the impact of human capital on longevity and health is unambiguously positive. ${ }^{11}$ Hence, the macro-level Mincerian relationship cannot hold: log-linearity is inevitably lost upon aggregation.

\section{Human capital accumulation and growth}

In the basic case discussed above, human capital accumulation cannot be the source of aggregate economic growth even if a linear schooling technology is assumed at the individual level. ${ }^{12}$ Let us now contrast this finding with a number of

\footnotetext{
${ }^{10}$ The proof uses the fact that $\int_{0}^{\infty} f(\tau) d \tau=1$. In the main step, we exploit the fact that both sides of (9) are identical functions of $\Phi$, so we can differentiate them with respect to $\Phi$ and equalize the derivatives.

${ }^{11}$ A survey of studies that confirm this effect can be found in Davies (2002), Section V. Furthermore, the question of accumulation of aggregate human capital when the survival law depends positively on human capital levels is a very important one. We leave it for future work.

${ }^{12}$ For empirical results in this field, see e.g. Benhabib and Spiegel (1994) or de la Fuente and Doménech (2006).
} 
reasons for which human capital accumulation may in fact contribute to economic growth.

1. Technological progress (generated outside of the human capital accumulation sector) may increase unit efficiencies of schooling and work effort. Thus, $\lambda(t)$ and $\mu(t)$ may be increasing over time. Then the average human capital level $\bar{h}(t)$ will rise over time, too, even in a society whose age structure is stationary. Moreover, since human capital is one of the factors of production, its increases will consequently contribute to aggregate economic growth. As Arias and McMahon (2001) show using US data, this mechanism is indeed very likely to work in reality. In such case, however, the human capital sector could only enhance or facilitate economic growth but not generate it. Its fundamental source is clearly not in human capital accumulation but in technological progress which makes $\lambda(t)$ and $\mu(t)$ grow over time.

2. Human capital at birth may be increasing over time. That is, $h(t, 0)$ may be considered an increasing function of time. There is no obvious reason for which $h(t, 0)$ could be growing over time, though. One could mention improved nutrition, natural selection, genetic engineering, etc., but neither of those explanations seems plausible or useful for explaining economic growth. Moreover, even if $h(t, 0)$ does grow over time, creation of economic growth is again external to human capital accumulation itself.

3. The demographic structure (age profile) of the society might change over time. In particular, ongoing increases in longevity and decreases in the birth rate should raise the average human capital level and thus render more growth - at least temporarily, though not necessarily in the long run. See Boucekkine, de la Croix and Licandro (2002, 2003) for an in-depth discussion of this issue. Faruqee (2003) is a further reference in this field.

4. Externalities in human capital accumulation may arise. Increments to the individual's human capital may be proportional to the average human capital level of her teachers and co-workers $\bar{h}(t+\tau)$ rather than to her own human capital level $h(t, \tau)$. Even more plausibly, increments to one's skills may be proportional to a certain aggregate value which includes both her own human capital $h(t, \tau)$ and the average human capital $\bar{h}(t+\tau)$ (e.g. a Cobb-Douglas bundle of the two). Voluminous literature in this field includes among others Ben-Porath (1967), Borjas (1992, 1995), Rangazas (2000, 2005), and Manuelli and Seshadri (2005).

Externalities in human capital accumulation are associated with knowledge spillovers and the complementarity between human capital and technology. 
They are clearly amongst the most promising explanations for the large role which is typically attributed to human capital in generating aggregate economic growth.

\section{Externalities}

Our main argument supporting the view that human capital accumulation may in some cases drive aggregate growth is based on external effects. We first note that it is often argued in literature that there exist substantial social returns to education on top of the fully appropriable private returns. These additional social returns could be due to knowledge spillovers, appearing at the level of family, local community (Rangazas, 2005), and the whole society. ${ }^{13}$ Standard estimates of the magnitude of private returns to an additional year of schooling fall in the range between $6 \%$ and $10 \%$ (see Card, 1999). ${ }^{14}$ Social returns are markedly more difficult to estimate. This strand of empirical literature has been surveyed by Davies (2002). A notable negative example is due to Acemoglu and Angrist (2000) who find negligibly small social returns to schooling. Davies (2002), on the other hand, concludes with a guess that "it is possible that education externalities could amount to something like $6-8 \%$ points."

Social returns to human capital accumulation are not only knowledge spillovers. It is argued that human capital speeds the adoption of new technologies and is strongly complementary to technology (Bils and Klenow, 2000). However, if technology adoption were the main channel of impact of human capital on growth, then the source of growth would be not human capital accumulation itself but technological progress. We shall thus concentrate on knowledge spillovers.

We incorporate externalities in the human capital accumulation technology as follows by assuming that the increments to individual human capital are no longer proportional to one's own human capital but to the average human capital in the society instead. With such technology, there exist a range of parameter values under which human capital accumulation drives long-run growth very much along the lines of the Uzawa-Lucas model. This is because, as opposed to individual human capital, average human capital is not embodied in any particular person; it may increase due to schooling and on-the-job training, and decrease due to births and deaths, but its overall evolution can go in either direction, depending on the

\footnotetext{
${ }^{13}$ Furthermore, there could be significant spatial concentration of knowledge spillovers in densely populated areas.

${ }^{14}$ Arias and McMahon (2001) argue that these estimates, based on cross-section studies, may be biased downwards due to the persistent upward trend in average earnings. Belzil and Hansen (2002) find that "contrary to conventional wisdom, the log wage regression is (...) convex in schooling". The same authors identify the "discount rate bias" which shifts the estimates upwards: those who obtain more schooling also experience higher average returns.
} 
relative strength of the forces at play. Even more plausibly, we shall consider a generalized case comprised between our original specification as given in (1) and the "fully external" case described above. Such case, put forward by Ben-Porath (1967) and strongly supported by Rangazas (2000) and Tamura (2001), has the increments to individual human capital proportional to a CRTS (constant returns to scale) Cobb-Douglas bundle of one's own and society's average human capital. The generalized case imposes more stringent conditions under which long-run balanced growth is possible but does not rule out this option. ${ }^{15}$

Let us now presume that schooling consists partly of transferring knowledge from teachers to pupils and partly of learning by the pupils themselves (cf. BenPorath, 1967). Increments to one's knowledge are assumed to be proportional to a CRTS Cobb-Douglas bundle of her own human capital $h(t, \tau)$ and the societywide average human capital embodied in her teachers, $\bar{h}(t)$. For ease of exposition, we disregard on-the-job training here $(\mu=0)$. The individual's human capital accumulation equation reads then:

$$
\frac{d}{d \tau} h(t, \tau)=\lambda \ell_{h}(\tau)[h(t, \tau)]^{\theta}[\bar{h}(t+\tau)]^{1-\theta}, \quad \theta \in[0,1) .
$$

Please note that the case $\theta=1$ corresponds to our original setup of Section 2 that ignores all externalities. The second polar case $\theta=0$, on the other hand, has so strong externalities that the role of individual human capital in its own accumulation is nil. It can be interpreted as an extreme case where education is exclusively about transferring knowledge from teachers to pupils and not at all about individual learning. We shall see shortly that the case $\theta=0$ offers especially transparent results which we treat as another benchmark in further comparisons.

Coupled with the initial condition $h(t, 0)=h_{0}>0$, equation (12) is readily solved as:

$$
h(t, \tau)=\left[(1-\theta) \lambda \int_{0}^{\tau} \ell_{h}(s)(\bar{h}(t+s))^{1-\theta} d s+h_{0}^{1-\theta}\right]^{\frac{1}{1-\theta}} .
$$

Using the "perpetual youth" survival law to obtain an integral equation that implicitly defines the aggregate human capital level $\bar{h}(t)$, we get:

$$
\bar{h}(t)=b \int_{0}^{\infty} e^{-b \tau}\left[(1-\theta) \lambda \int_{0}^{\tau} \ell_{h}(s)(\bar{h}(t-\tau+s))^{1-\theta} d s+h_{0}^{1-\theta}\right]^{\frac{1}{1-\theta}} d \tau .
$$

\footnotetext{
${ }^{15}$ We have also checked what happens if teachers are systematically better educated than pupils and if education consists primarily in transferring teachers' knowledge to pupils. It turns out that aggregate growth is not possible in such case. This case is presented in Section 6.
} 
To check if this formulation can give rise to balanced growth in $\bar{h}(t)$, at least asymptotically, we proceed as follows. We insert an exponential solution $\bar{h}(t)=$ $\bar{h}_{0} e^{\beta t}$ into (14) and calculate the integrals under the assumption $\ell_{h}=\bar{\ell}_{h} \equiv$ const (which we make for tractability). It turns out that the current case is in certain cases consistent with balanced growth, but this result depends crucially on the magnitude of knowledge spillovers, as measured by $1-\theta$, and on the relative efficiency of education $\lambda \bar{\ell}_{h}$ as compared to the birth rate $b$. Working with the limit $t \rightarrow \infty$ we obtain the following proposition.

Proposition 1 Assume $\theta \in[0,1)$ and $\bar{\ell}_{h} \equiv$ const. There exists a unique asymptotic balanced growth path that has aggregate human capital $\bar{h}(t)$ growing exponentially over time with a growth rate $\beta>0$ if and only if

$$
\lambda \bar{\ell}_{h}>\frac{b}{(1-\theta)\left(\Gamma\left(\frac{2-\theta}{1-\theta}\right)\right)^{1-\theta}} .
$$

If the sign in (15) is reversed then there exists a unique asymptotic steady state $\bar{h}_{0}$ where aggregate human capital is constant over time.

Proof. Insert an exponential solution $\bar{h}(t)=\bar{h}_{0} e^{\beta t}$ into (14). It is obtained that

$$
\bar{h}_{0} e^{\beta t}=b \int_{0}^{\infty} e^{-b \tau}\left[\frac{\lambda \ell_{h}}{\beta} \bar{h}_{0}^{1-\theta} e^{-\beta(1-\theta) t}\left(1-e^{-\beta(1-\theta) \tau}\right)+h_{0}^{1-\theta}\right]^{\frac{1}{1-\theta}} d \tau .
$$

Case $\beta>0$. In such case, we can divide (16) sidewise by $\bar{h}_{0} e^{\beta t}$ and take the limit $t \rightarrow \infty$ so that the term with $h_{0}$ disappears. The following characteristic equation is obtained:

$$
\Psi(\beta)=1-b\left(\lambda \bar{\ell}_{h}\right)^{\frac{1}{1-\theta}} \int_{0}^{\infty} e^{-b \tau}\left(\frac{1-e^{-\beta(1-\theta) \tau}}{\beta}\right)^{\frac{1}{1-\theta}} d \tau=0 .
$$

We will show that $\Psi(\beta)$ crosses zero exactly once in its domain $\beta>0$ (and thus a unique growth rate $\beta$ exists) if and only if (15) is satisfied. First, note that $\lim _{\beta \rightarrow \infty} \Psi(\beta)=1>0$. Second, calculate the derivative $\Psi^{\prime}(\beta)$ :

$$
\begin{aligned}
\Psi^{\prime}(\beta) & =-b\left(\lambda \bar{\ell}_{h}\right)^{\frac{1}{1-\theta}} \times \\
& \times \int_{0}^{\infty} e^{-b \tau} \frac{1}{1-\theta}\left(\frac{1-e^{-\beta(1-\theta) \tau}}{\beta}\right)^{\frac{\theta}{1-\theta}} \frac{e^{-\beta(1-\theta) \tau}(1+\beta(1-\theta) \tau)-1}{\beta^{2}} d \tau .
\end{aligned}
$$

All factors of the derivative can be unambiguously signed positive but for the minus in front of the expression and the factor $e^{-\beta(1-\theta) \tau}(1+\beta(1-\theta) \tau)-1$ which is negative since $e^{x}>1+x$ for all $x \neq 0$. We can thus conclude that $\Psi^{\prime}(\beta)>0$ 
so $\Psi$ is increasing in its whole positive domain. Hence, a unique $\beta>0$ such that $\Psi(\beta)=0$ exists if and only if $\lim _{\beta \rightarrow 0_{+}} \Psi(\beta)<0$. From de l'Hospital's rule we obtain $\lim _{\beta \rightarrow 0_{+}} \frac{1-e^{-\beta(1-\theta) \tau}}{\beta}=(1-\theta) \tau$. Finally:

$$
\begin{aligned}
\lim _{\beta \rightarrow 0_{+}} \Psi(\beta) & =1-b\left[(1-\theta) \lambda \bar{\ell}_{h}\right]^{\frac{1}{1-\theta}} \int_{0}^{\infty} e^{-b \tau} \tau^{\frac{1}{1-\theta}} d \tau \\
& =1-\left[\frac{(1-\theta) \lambda \bar{\ell}_{h}}{b}\right]^{\frac{1}{1-\theta}} \Gamma\left(\frac{2-\theta}{1-\theta}\right) .
\end{aligned}
$$

Rearranging this slightly, we get $\lim _{\beta \rightarrow 0_{+}} \Psi(\beta)<0 \Leftrightarrow \lambda \bar{\ell}_{h}>\frac{b}{(1-\theta)\left(\Gamma\left(\frac{2-\theta}{1-\theta}\right)\right)^{1-\theta}}$.

Case $\beta<0$. In such case, taking the limits as $t \rightarrow \infty$ in (16) sidewise yields $0=b \int_{0}^{\infty} e^{-b \tau} h_{0} d \tau=h_{0}$ which contradicts the assumption $h_{0}>0$. Such case is thus impossible.

Case $\beta=0$. Inserting $\bar{h}(t) \equiv \bar{h}_{0}$ into (14) and dividing sidewise by $\bar{h}_{0}$, we obtain

$$
\Xi\left(\bar{h}_{0}\right)=b \int_{0}^{\infty} e^{-b \tau}\left[(1-\theta) \lambda \bar{\ell}_{h} \tau+\left(\frac{h_{0}}{\bar{h}_{0}}\right)^{1-\theta}\right]^{\frac{1}{1-\theta}} d \tau-1=0 .
$$

We will now show that a unique solution $\bar{h}_{0} \geq h_{0}$ to this equation exists if and only if $\lambda \bar{\ell}_{h}<\frac{b}{(1-\theta)\left(\Gamma\left(\frac{2-\theta}{1-\theta}\right)\right)^{1-\theta}}$. First, it is easy to see that

$$
\Xi\left(h_{0}\right)=b \int_{0}^{\infty} e^{-b \tau}\left[(1-\theta) \lambda \bar{\ell}_{h} \tau+1\right]^{\frac{1}{1-\theta}} d \tau-1>b \int_{0}^{\infty} e^{-b \tau} d \tau-1=0 .
$$

Second, we calculate the derivative of $\Xi$ :

$$
\Xi^{\prime}\left(\bar{h}_{0}\right)=-b \int_{0}^{\infty} e^{-b \tau}\left[(1-\theta) \lambda \bar{\ell}_{h} \tau+\left(\frac{h_{0}}{\bar{h}_{0}}\right)^{1-\theta}\right]^{\frac{\theta}{1-\theta}} d \tau \cdot \frac{h_{0}^{1-\theta}}{\bar{h}_{0}^{2-\theta}}<0
$$

Then, there exists a unique $\bar{h}_{0}$ such that $\Xi\left(\bar{h}_{0}\right)=0$ if and only if $\lim _{\bar{h}_{0} \rightarrow \infty} \Xi\left(\bar{h}_{0}\right)<$ 0 . Analogously to the calculations above, we obtain:

$$
\lim _{\bar{h}_{0} \rightarrow \infty} \Xi\left(\bar{h}_{0}\right)=\left[\frac{(1-\theta) \lambda \bar{\ell}_{h}}{b}\right]^{\frac{1}{1-\theta}} \Gamma\left(\frac{2-\theta}{1-\theta}\right)-1 .
$$

Rearranging this expression, we find that $\lim _{\bar{h}_{0} \rightarrow \infty} \Xi\left(\bar{h}_{0}\right)$ is negative if and only if $\lambda \bar{\ell}_{h}<\frac{b}{(1-\theta)\left(\Gamma\left(\frac{2-\theta}{1-\theta}\right)\right)^{1-\theta}}$ which completes the proof.

Several things should be emphasized here. First, the characteristic equation (17) reduces to $\beta=\lambda \bar{\ell}_{h}-b$ for $\theta=0$ : the unique growth rate $\beta$ is positive if 
$\lambda \bar{\ell}_{h}>b$ - i.e. if the pace of learning outruns the flow of births (people are born uneducated and the role of deaths is nil in the perpetual youth case, cf. Faruqee, 2003). If the flow of births diluting average human capital is greater than the pace of human capital accumulation through schooling, aggregate human capital will stagnate. More generally, we observe that because of natural depreciation the long-run growth rate is uniformly lower than the instantaneous rate of human capital formation through schooling $\left(\lambda \bar{\ell}_{h}\right)$ :

Proposition 2 The balanced growth rate $\beta$ is always lower than $\lambda \bar{\ell}_{h}$.

Proof. If $\beta=0$ then trivially $\beta<\lambda \bar{\ell}_{h}$. Assume in turn $\beta>0$. In such case, from (18) we know that $\Psi^{\prime}(\beta)>0$. To show that $\Psi(\beta)=0$ for $\beta<\lambda \bar{\ell}_{h}$, it suffices then to show $\Psi\left(\lambda \bar{\ell}_{h}\right)>0$. This inequality holds since

$$
\Psi\left(\lambda \bar{\ell}_{h}\right)=1-b \int_{0}^{\infty} e^{-b \tau}\left(1-e^{-\beta(1-\theta) \tau}\right)^{\frac{1}{1-\theta}} d \tau>1-b \int_{0}^{\infty} e^{-b \tau} d \tau=1-1=0
$$

Second, one could say that in a sense, by assuming strong society-level spillovers $1-\theta$ or a very high schooling intensity $\lambda \bar{\ell}_{h}-$ as required by $(15)$ - in fact we construct a "vintage Uzawa-Lucas model": human capital is accumulated according to a linear technology (using a CRTS bundle of the individual or the aggregate human capital) and is subject to depreciation with a constant rate $b$. In effect, our microfounded aggregate human capital accumulation equation resembles, at least in the asymptotic BGP approximation, the Uzawa-Lucas equation $\dot{\bar{h}}(t)=\beta \bar{h}(t)$ which gives rise to long-run balanced growth as long as $\beta>0$. Furthermore, if $\theta=0$ then $\beta=\lambda \bar{\ell}_{h}-b$ and thus the growth rate is directly the difference between the unit efficiency of education and the birth rate. However, the case $\theta=0$ looks empirically implausible (Rangazas, 2000; Tamura, 2001; Manuelli and Seshadri, 2005). Instead, there is evidence that $\theta$ is substantially larger, preferably falling in the range $(0.75,0.8)$ : see Borjas $(1992 ; 1995)$ and Rangazas (2000). If $\theta=0.75$, then for balanced growth it is required - according to $(15)$ - that $\lambda \bar{\ell}_{h}>1.8072 b$; if $\theta=0.8$, the condition becomes $\lambda \bar{\ell}_{h}>1.9193 b$.

Third, if (15) does not hold, then the role of human capital at birth $\left(h_{0}\right)$ is not negligible in the long run. On the contrary, we find that a unique asymptotic steady state $\bar{h}_{0}$ exists then and that the steady-state aggregate human capital level depends on $h_{0}$. Even more precisely, we find that $h_{0}>0$ imposes a lower bound on the aggregate human capital level and rules out its exponential decline even though in such case, the characteristic equation (17) has a negative root.

Fourth, the lower bound imposed on schooling intensity by the balanced growth requirement (the right-hand side of (15)) depends on the birth rate $b$ positively and linearly, so that the greater the birth rate, the less viable is longrun growth driven by aggregate human capital accumulation. We also find its 
positive dependence on $\theta$ - i.e. negative dependence on the magnitude of knowledge spillovers. The factor multiplying $b$ on the right-hand side of (15) rises smoothly from 1 when $\theta=0$ to $e$ when $\theta \rightarrow 1_{-}$. This result is also intuitive: the stronger are spillovers in education, the greater the opportunities for balanced growth (and the faster is growth, as we shall prove below).

Apart from the special case $\theta=0$ where $\beta=\lambda \bar{\ell}_{h}-b$, the growth rate of aggregate human capital cannot be explicitly calculated: the characteristic equation (17) does not offer explicit formulas. However, we can still infer the direction of dependence between $\beta$ and crucial parameters of the model. The following proposition holds.

Proposition 3 The asymptotic balanced growth rate $\beta$ depends positively on effective schooling intensity $\lambda \bar{\ell}_{h}$ and negatively on the birth rate $b$ and the share of one's own human capital in the schooling technology, $\theta$.

Analogous calculations may be carried out for the level of aggregate human capital at the asymptotic steady state $\bar{h}_{0}$ if balanced growth is ruled out. We obtain the following results:

Proposition 4 The level of aggregate human capital at the asymptotic steady state $\bar{h}_{0}$ depends positively on effective schooling intensity $\lambda \bar{\ell}_{h}$ and human capital at birth $h_{0}$, but negatively on the birth rate $b$ and the share of one's own human capital in the schooling technology, $\theta$.

All results summarized in the propositions above conform with our initial intuitions: greater schooling intensity $\lambda \bar{\ell}_{h}$ has an unambiguously positive impact either on the long-run growth rate of the economy (if growth is feasible) or on the steady-state human capital level (if growth is not feasible). This positive impact, present already in the short run, carries forward to the long run without any disturbances. The same reasoning can be applied to the birth rate $b$, responsible for the depreciation of aggregate human capital. Furthermore, stronger spillovers imply more growth (or a higher steady-state level of human capital) because they reduce the role of replacement investment: while individual human capital is embodied and thus lost upon death, aggregate human capital has the disembodied character and "lives on" despite deaths and births.

\section{The Uzawa-Lucas model revisited}

Let us now discuss one scenario that is not feasible when finiteness of people's lives and the embodied character of human capital are properly accounted for. Namely, human capital accumulation cannot give rise to endogenous balanced 
economic growth along the lines of the Uzawa-Lucas model (Uzawa, 1965; Lucas, 1988, 1993; Barro and Sala-i-Martin, 1995, Chapter 5; Gong, Greiner, and Semmler, 2004). The main reason is that by assuming the representative agent to be infinitely-lived (or under an alternative interpretation, by assuming human capital to be disembodied), this model ignores the natural depreciation of aggregate human capital due to births and deaths as well as replacement investment in education. To put it shortly, the basic Uzawa-Lucas model is just not compatible with intergenerational aggregation of human capital.

The assumptions of the Uzawa-Lucas model may be, however, successfully defended using the concept of knowledge spillovers. To reconcile the model with depreciation of aggregate human capital $\bar{h}$, first the condition (15) that the constant unit intensity of schooling $\lambda \bar{\ell}_{h}$ is high enough to overweigh depreciation must be satisfied. The crux of the argument is then that the linear production function of form $\dot{\bar{h}}=B \bar{h}$ where $B>0$, postulated in the Uzawa-Lucas model, can be interpreted as $\dot{\bar{h}}=A \bar{h}-\delta \bar{h}$ where $A>\delta$, and $\delta$ is the human capital depreciation rate which must be positive due to births and deaths. Such linear production function at the aggregate level requires strong society-level knowledge spillovers, though, as we have just shown in Section 4 above. Otherwise, the marginal product of human capital would gradually fall down to zero instead of remaining constant.

A different treatment of human capital accumulation can be found in the classic work of Mankiw, Romer, and Weil (1992). Their macro approach accounts neither for the embodied character of human capital nor for finite lifespans of people, but it includes depreciation of aggregate human capital which, in the light of the above discussion, could be argued to capture the effect of births and deaths. Moreover, this neoclassical model converges to a steady state where aggregate human capital is constant, just like in our basic aggregation exercise of Section 2 or in a case where spillovers are allowed for but yet equation (15) is violated.

\section{Extensions}

Until this point we have shown that if the vintage structure of human capital is properly accounted for, and after the aggregation procedure has been carried out, (i) the Mincer equation does not hold at the macro level even if it holds at the micro level, and (ii) human capital accumulation cannot be the source of balanced growth unless there are substantial knowledge spillovers involved. Let us now provide a few qualifications to these arguments. 


\subsection{Removing the linearity at the individual level}

Assuming that the increments to one's human capital are more than proportional to her actual human capital level implies that there exists a finite age at which the individual's human capital reaches infinity (assuming that she has not quit school earlier). On the one hand, such educational "nirvana" seems highly implausible, but on the other hand, one must note that when there are only mildly increasing returns to human capital accumulation, the age at which human capital explodes to infinity might be very large, e.g. such that under plausible survival laws, nobody would ever live up to such age. And more importantly, recent empirical findings by Belzil and Hansen (2002) support the increasing-returns case: they find log wages to be convex in schooling at the micro level.

The converse assumption that at the individual level, increases in human capital are less than proportional to actual human capital levels, does not cause such explosivity problems, especially painful when one sticks to the "perpetual youth" survival law which imposes no upper bound on people's age. ${ }^{16}$

The current generalization of equation (1) reads:

$$
\frac{d}{d \tau} h(t, \tau)=\left(\lambda \ell_{h}(\tau)+\mu \ell_{Y}(\tau)\right) h^{\phi}(t, \tau), \quad \phi>0, \quad \phi \neq 1
$$

with $h(t, 0)=h_{0}>0$. Solving this leads to a micro-level cross-section equation human capital regressed on schooling effort and work experience - which takes a hyperbolic form instead of the exponential one:

$$
h(t, \tau)=\left[h_{0}^{1-\phi}+(1-\phi)\left(\lambda \int_{0}^{\tau} \ell_{h}(s) d s+\mu \int_{0}^{\tau} \ell_{Y}(s) d s\right)\right]^{\frac{1}{1-\phi}} .
$$

The original exponential Mincer equation is obtained only in the knife-edge case of $\phi=1$. Even more precisely, if we assume that an individual of age $\tau$ first engages in full-time education $\left(\ell_{h}=1\right)$ for time $\tau_{h}$ and then works full-time $\left(\ell_{Y}=1\right)$ for time $\tau_{Y}$, with $\tau_{h}+\tau_{Y} \leq \tau$, we obtain the following alternative to the Mincerian specification:

$$
h^{1-\phi}=h_{0}^{1-\phi}+(1-\phi)\left(\lambda \tau_{h}+\mu \tau_{Y}\right)
$$

In principle, this result does not have to be troublesome: if in reality $\phi \approx 1$, the true hyperbolic relationship is approximated by the exponential one quite well. No significant error should be expected then if the exponential one is estimated

\footnotetext{
${ }^{16}$ Decreasing returns in human capital accumulation have been found by Psacharopoulos (1994) as well as Bils and Klenow (2000), but at the macro level of countries so their results are not directly applicable here.
} 
instead. If, however, marked departures from the constant-returns case are expected, signifying that $\phi \neq 1$ is robust, then the hyperbolic equation (26) should be preferred. In particular, if $\phi>1$ then increasing returns in human capital accumulation are observed, and log wages are convex in schooling like in Belzil and Hansen (2002) and Figure 2. Conversely, if $\phi<1$ then decreasing returns in human capital accumulation prevail, and log wages are concave in schooling.

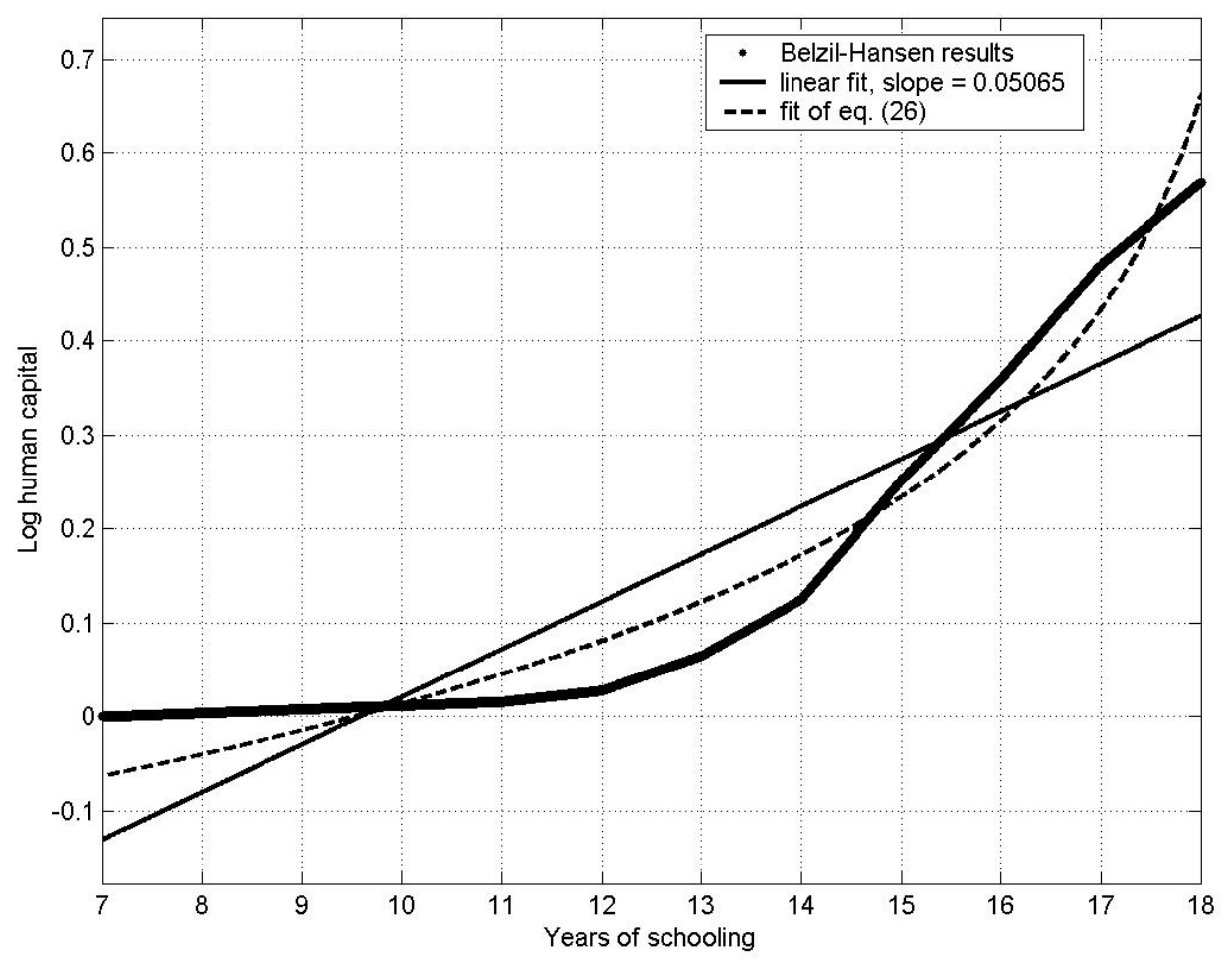

Figure 2: The micro-level study by Belzil and Hansen (2002) suggests that log human capital is generally convex in years of schooling. Their results can be approximated by an explosive equation (26) with $\phi=4.873, \lambda=0.0281$, and $h_{0}=0.0635$ (robust NLS estimates), yielding $R^{2}=0.95$. The log-linear OLS fit with a constant rate of return equal to 0.05065 is significantly worse $\left(R^{2}=0.775\right)$.

We also remark that since $h(t, \tau)$ does not depend on $t$ here, average human capital in a society whose age structure is stationary cannot change over time.

Summing up, if the linearity at the individual level is removed, then the Mincer earnings equation fails to hold not only at the macro level of countries, but also at the micro level of individuals. Furthermore, human capital accumulation without externalities cannot drive aggregate growth even if there are increasing returns at 
the individual level because human capital is embodied in people and lost upon death.

\subsection{More realistic survival laws}

Let us now return to the linear specification (1) but move away from the unrealistic "perpetual youth" survival law, exploited throughout the paper. In reality, youth is of course "fleeting" rather than perpetual (Faruqee, 2003) and an upper bound on people's lifespans exists. We shall see shortly that this change in the treatment of demographics does not overturn our main results.

First, remind that a stationary age structure and a steady death rate $d^{*}$ are compatible with any survival law $m(\tau)$. We only have to ensure that $d^{*}$ solves the implicit equation (7). For simplicity, we concentrate exclusively on stationary age structures here.

As an representative example, we use the realistic survival law put forward by Boucekkine, de la Croix, and Licandro (2002). These authors let the unconditional probability of reaching an age of $\tau$ be given by

$$
m(\tau)= \begin{cases}\frac{\alpha-e^{\beta \tau}}{\alpha-1}, & \tau \leq \frac{\ln \alpha}{\beta} \\ 0, & \tau>\frac{\ln \alpha}{\beta} .\end{cases}
$$

It is assumed that $\alpha>1$ and $\beta>0$. This survival law imposes an upper bound on people's lifespans: nobody can live longer than $M \equiv(\ln \alpha) / \beta$ years. The steady-state death rate $d^{*}$ solves the implicit equation

$$
d=\frac{\beta b}{\alpha-1}\left(\frac{\alpha^{\frac{\beta-b+d}{\beta}}-1}{\beta-b+d}\right),
$$

which offers two roots $d^{*}$. The first one is the trivial root $d^{*}=b$ that implies a constant population. The second one implies either a growing or a declining population: population will grow if

$$
b E>1 \Leftrightarrow \frac{b(\alpha \ln \alpha-\alpha+1)}{\beta(\alpha-1)}>1,
$$

and decline if $b E<1$. If $b E=1$, then the trivial root $d^{*}=b$ is unique.

We shall assume $\ell_{h}=\bar{\ell}_{h} \equiv$ const as well as $\ell_{Y}=\bar{\ell}_{Y} \equiv$ const for simplicity, and denote $\Phi=\lambda \bar{\ell}_{h}+\mu \bar{\ell}_{Y}$. Aggregating the individual levels of human capital 
(2) over the whole age structure of the society, we get

$$
\begin{aligned}
\bar{h}(t) & =\int_{0}^{\infty} b e^{-(b-d) \tau} m(\tau) h(t-\tau, \tau) d \tau= \\
& =\frac{b h_{0}}{\alpha-1}\left[\alpha \frac{e^{(\Phi-b+d) M}-1}{\Phi-b+d}-\frac{e^{(\Phi-b+d+\beta) M}-1}{\Phi-b+d+\beta}\right]= \\
& =b h_{0}\left[\frac{\alpha \beta}{(\Phi-b+d+\beta)(\Phi-b+d)}\left(\frac{\alpha^{\frac{\Phi-b+d}{\beta}}-1}{\alpha-1}\right)-\frac{1}{\Phi-b+d+\beta}\right] .
\end{aligned}
$$

As expected, equation (30) is quite different from the hyperbolic equation (6) but it is not the Mincer equation either, so that the Mincerian log-linear earnings-schooling relationship is again lost upon aggregation. ${ }^{17}$ Of course, we also find that $\bar{h}(t)$ is independent of $t$ so that human capital accumulation cannot be the engine of growth in the absence of knowledge spillovers. In sum, our two basic results go through also in the case of a more realistic survival law which implies that youth is fleeting.

\subsection{Time profiles of education and work effort}

One further criticism could be raised that we have been using simplistic assumptions that either (i) $\ell_{h}$ and $\ell_{Y}$ are constant over time, or (ii) the individuals first attend school full time, and then work full time. These assumptions simplify the algebra greatly but are not conducive for our main results, though.

Let us provide an illustrative example for this claim. Assume that at the individual level, human capital is accumulated according to the linear equation (1), without any externalities from the average human capital level. Suppose further that all time is split between schooling and work only, $\ell_{h}(\tau)+\ell_{Y}(\tau)=1$ for all ages $\tau$.

We shall posit a particular time profile of age-specific learning effort: at the age $\tau$, the individual assigns to formal schooling a share of time $\ell_{h}(\tau)=\theta /(\tau+$ $\left.\theta^{1 / \psi}\right)^{\psi}$, with $\theta>0$ and $\psi \in(0,1)$. This implies that $\ell_{h}(0)=1$ and from then on, learning effort declines according to a power law, gradually giving way to working: an individual aged $\tau$ devotes a share of time $\ell_{Y}(\tau)=1-\theta /\left(\tau+\theta^{1 / \psi}\right)^{\psi}$ to working.

\footnotetext{
${ }^{17}$ However, in the current case the log earnings vs years of schooling relationship is numerically quite close to linear. This finding follows from an exercise using Boucekkine, de la Croix, and Licandro's (2003) estimates $\alpha=5.4365$ and $\beta=0.01472$, together with $b=d=0.02$ that yield a stable population, and $h_{0}=0.0635 ; \lambda=0.0281$ which are robust NLS estimates of the micro Mincer equation based on the results of Belzil and Hansen (2002). Although the resultant curve is not linear but convex, the macro Mincer equation with slope 0.05062 fits equation (30) almost perfectly, with $R^{2}=0.99845$.
} 
Equation (2) is still obtained and it still means that log human capital depends linearly on total schooling effort and total work effort - the relevant integrals denote these cumulative measures. After integration, it is then obtained that

$$
h(t, \tau)=h_{0} \exp \left\{\frac{(\lambda-\mu) \theta}{1-\psi}\left(\tau+\theta^{\frac{1}{\psi}}\right)^{1-\psi}+\tau-\frac{\lambda-\mu}{1-\psi} \theta^{\frac{1}{\psi}}\right\} .
$$

One obvious observation is that without externalities, individuals' human capital $h(t, \tau)$ does not depend on $t$ and thus, $\bar{h}(t)$ is constant over time.

The second observation is slightly more surprising. The expression in the exponent of equation (31) above is linear in lifelong schooling effort, but it follows a concave shape in raw years of schooling.

It is clear that in real data, one finds variables like "years of schooling" rather than "cumulative lifelong schooling effort" which lies at the foundation of our theoretical considerations. These two variables are related but not functionally dependent. Indeed, in our example we could presume that the individual "leaves school" when her instantaneous schooling effort falls below a certain threshold, say $1 / n$. This happens at the age $\tau_{h}^{*}=\theta^{1 / \psi}\left(n^{1 / \psi}-1\right)$ which is proportional to $\theta^{1 / \psi}$ and $n$ just fixes the coefficient of proportionality. Then, the "schooling part" of the exponent in (31) can be written as

$$
\frac{\lambda}{(1-\psi)\left(n^{1 / \psi}-1\right)}\left\{\left(\left(n^{1 / \psi}-1\right) \tau\left(\tau_{h}^{*}\right)^{\frac{\psi}{1-\psi}}+\left(\tau_{h}^{*}\right)^{\frac{1}{1-\psi}}\right)^{1-\psi}-\tau_{h}^{*}\right\},
$$

which is concave in $\tau_{h}^{*}$.

The conclusion is that the Mincerian result calls for a log-linear pattern of dependence between human capital and total schooling effort. The latter variable may not be captured properly in the data on years of schooling, however, if the time profile of learning is smooth and people start working when still at school or don't stop learning immediately after graduation. In such cases, a concave relationship between log human capital and years of schooling should be expected, presumably following the lines of Bils and Klenow (2000).

\subsection{The case of teachers having proportionally more hu- man capital}

Let us now presume that formal education consists primarily in transferring knowledge from teachers to pupils and not in individual learning of the pupils. Suppose furthermore that each pupil is taught by teachers who are older than 
her by a constant number of years, say $\omega \cdot{ }^{18}$ Equation (1) is then replaced by

$$
\frac{d}{d \tau} h(t, \tau)=\lambda \ell_{h}(\tau) h(t, \tau+\omega)+\mu \ell_{Y}(\tau) h(t, \tau)
$$

Under the assumptions that $\ell_{h}=\bar{\ell}_{h} \equiv$ const as well as $\ell_{Y}=\bar{\ell}_{Y} \equiv$ const, equation (33) has one or two positive real roots (growth rates of individual human capital) if $\omega$ is low enough, precisely if

$$
\omega \mu \bar{\ell}_{Y}+\ln \omega+\ln \left(\lambda \bar{\ell}_{h}\right)+1 \leq 0
$$

In particular, there are exactly two real positive solutions if the left-hand side of (34) is strictly negative. If (34) is violated, however, then (33) is not consistent with exponential growth and cannot be used to generate balanced growth of personal human capital as the individual ages.

Much more light can be shed on the solution to equation (33) presupposing that (34) holds and assuming balanced growth in individual human capital: we can exploit the exponentiality property and replace the assumption that the teacher is $\omega$ years older than the pupil with the assumption that the teacher has proportionally more human capital. Mathematically, this means replacing $h(t, \tau+\omega)$ with $m h(t, \tau)$, where $m>1 .^{19}$

Using this "trick", equation (33) is quickly solved as

$$
h(t, \tau)=h_{0} \exp \left(\left(m \lambda \bar{\ell}_{h}+\mu \bar{\ell}_{Y}\right) \tau\right),
$$

which implies only a "cosmetic" modification to (2) and the cross-sectional Mincerian relationship is preserved. As $h(t, \tau)$ does not depend on $t$, we again conclude that the average level of human capital in the society will stay constant over time. Since we have assumed out externalities from aggregate human capital $(\bar{h}(t))$ here, current case cannot be used to generate balanced growth in aggregate human capital.

\section{Concluding remarks}

In this paper, we have carried out the aggregation of human capital across individuals taking into account the explicit vintage structure of the society and

\footnotetext{
${ }^{18}$ This assumption is not far fetched: it represents the idea that the subjects taught at elementary school do not require the knowledge necessary for lecturing at a university. The knowledge effectively transferred to a pupil is related to the kid's current grade at school rather than to the average human capital level in the society.

${ }^{19}$ It can be shown that $\omega>0$ and $m>1$ are related by the implicit equation $\omega\left(m \lambda \bar{\ell}_{h}+\mu \bar{\ell}_{Y}\right)-$ $\ln m=0$. We confirm that there exist two positive solutions $m_{1}(\omega)$ and $m_{2}(\omega)$ as long as $(34)$ is satisfied with strict inequality and one positive solution if (34) is satisfied with equality.
} 
the fact that differences in age necessarily imply heterogeneity in human capital levels.

Intergenerational heterogeneity in human capital, coupled with the fact that human capital is embodied in people whose lifespan is finite has far-reaching consequences both for economic growth theory and for the associated empirical literature, several of whose have in general not been acknowledged:

(i) the age structure of the society has an impact on the pattern of dependence between aggregate human capital and average years of schooling. If human capital accumulation at the level of individuals follows a linear technology then the well-documented (cf. Heckman, Lochner, and Todd, 2003) crosssectional Mincer equation is supposed to hold. It cannot hold at the macro level of countries, though. The log-linear relationship between human capital and schooling is thus inevitably lost upon aggregation;

(ii) human capital accumulation cannot drive aggregate growth unless we allow for strong society-level externalities (knowledge spillovers) in schooling. Standard growth-theoretic approaches (e.g. the Uzawa-Lucas model) typically fail to account for the depreciation of aggregate human capital due to deaths of the human-capital-rich and births of the human-capital-poor, and for the fact that substantial replacement investment in human capital has to be made before net investment is possible.

We have also provided a number of qualifications and robustness checks to our main argument and shown that it is not sensitive to relaxing some of our admittedly unrealistic assumptions.

A strong suggestion for further empirical research is then to pay special attention to the demographic structure of the population whose human capital is aggregated. Due to aggregation problems, the Mincer equation should not be expected to hold at the macro level of countries (cf. Krueger and Lindahl, 2001). Also, one should take additional care of the possibility that externalities in the form of knowledge spillovers may distort the Mincerian relationship also at the micro level.

In further theoretical work, the consequences of dynamic changes in the demographic structure (cf. Boucekkine, de la Croix, and Licandro, 2003) for the aggregate human capital level should be discussed. Another suggestion is to allow our survival laws to depend positively on the level of individual human capital. Finally, one could try to assess the roles of technological progress, human capital accumulation, and technology-skill complementarity in generating growth under embodied human capital with an explicit vintage structure.

Above all, however, three simple facts should never be disregarded in human capital theory: (i) that human capital is embodied in people, (ii) that people 
differ in age, and (iii) that in the end, each of us is going to die.

\section{References}

[1] Acemoglu, D., and Angrist, J. (2000) How large are human capital externalities? Evidence from compulsory schooling laws. NBER Macroeconomics Annual 2000, 9-58.

[2] Arias, O., and McMahon, W.W. (2001) Dynamic rates of return to education in the U.S. Economics of Education Review 20, 121-138.

[3] Barro, R.J., and Sala-i-Martin, X. (1995) Economic Growth. McGraw-Hill, Inc.

[4] Belzil, Ch., and Hansen, J. (2002) Unobserved ability and the return to schooling. Econometrica 70(5), 2075-2091.

[5] Benhabib, J., and Spiegel, M. (1994) The role of human capital in economic development: evidence from aggregate cross-country data. Journal of Monetary Economics 34(2), 143-173.

[6] Ben-Porath, Y. (1967) The production of human capital and the life cycle of earnings. Journal of Political Economy 75, 352-365.

[7] Bils, M., and Klenow, P. (2000) Does schooling cause growth? American Economic Review 90(5), 1160-1183.

[8] Blanchard, O. (1985) Debt, deficits, and finite horizons. Journal of Political Economy 93, 223-247.

[9] Bloom, D.E., Canning, D., and Sevilla, J. (2001) Health, human capital, and economic growth. Commision on Macroeconomics and Health Working Paper WG1:8.

[10] Borjas, G.J. (1992) Ethnic capital and intergenerational mobility. Quarterly Journal of Economics 107, 123-150.

[11] Borjas, G.J. (1995) Ethnicity, neighborhoods, and human-capital externalities. American Economic Review 85, 365-390.

[12] Boucekkine, R., de la Croix, D., and Licandro, O. (2002) Vintage human capital, demographic trends and endogenous growth. Journal of Economic Theory $104,340-375$. 
[13] Boucekkine, R., de la Croix, D., and Licandro, O. (2003) Early mortality declines at the dawn of modern growth. Scandinavian Journal of Economics $105,401-418$.

[14] Card, D.E. (1999) The causal effect of education on earnings. In: Ashenfelter, O., Card, D.E. (eds.) Handbook of labor economics Vol. 3, 1801-1863. Amsterdam: Elsevier.

[15] Davies, J. (2002) Empirical evidence on human capital externalities. University of Western Ontario, mimeo.

[16] de la Fuente, A., and Doménech, R. (2006) Human capital in growth regressions: how much difference does data quality make?, Journal of the European Economic Association 4(1), 1-36.

[17] Faruqee, H. (2003) Debt, deficits, and age-specific mortality. Review of Economic Dynamics 6, 300-312.

[18] Gong, G., Greiner, A., and Semmler, W. (2004) The Uzawa-Lucas model without scale effects: theory and empirical evidence, Structural Change and Economic Dynamics 15, 401-420.

[19] Hall, R.E., and Jones, C.I. (1999) Why do some countries produce so much more output per worker than others? Quarterly Journal of Economics 114(1), 83-116.

[20] Heckman, J., and Klenow, P. (1997) Human capital policy. University of Chicago, mimeo.

[21] Heckman, J., Lochner, L.J., and Todd, P.E. (2003) Fifty years of Mincer earnings regressions. NBER Working Paper 9732.

[22] Jones, C.I. (2005) Growth and ideas. [In:] P. Aghion and S.N. Durlauf, eds., Handbook of Economic Growth. Amsterdam: North-Holland.

[23] Krueger, A., and Lindahl, M. (2001) Education for growth. Why and for whom? Journal of Economic Literature 39(4), 1101-1136.

[24] Lucas, R.E. (1988) On the mechanics of economic development. Journal of Monetary Economics 22(1), 3-42.

[25] Lucas, R.E. (1993) Making a miracle. Econometrica 61(2), 251-272.

[26] Mankiw, N.G., Romer, D., and Weil, D.N. (1992) A contribution to the empirics of economic growth. Quarterly Journal of Economics 107(2), 407-437. 
[27] Manuelli, R.E., and Seshadri, A. (2005) Human capital and the wealth of nations. University of Wisconsin-Madison, mimeo.

[28] Mincer, J. (1958) Investment in human capital and personal income distribution. Journal of Political Economy 66(4), 281-302.

[29] Mincer, J. (1974) Schooling, Experience, and Earnings. New York: Columbia University Press.

[30] Pandey, M. (2005) Human capital aggregation and relative wages across countries. University of Winnipeg, mimeo.

[31] Psacharopoulos, G. (1994) Returns to investment in education: a global update. World Development 22(9), 1325-1343.

[32] Rangazas, P.C. (2000) Schooling and economic growth: a King-Rebelo experiment with human capital. Journal of Monetary Economics 46, 397-416.

[33] Rangazas, P.C. (2005) Human capital and growth: an alternative accounting. Topics in Macroeconomics 5(1), Art. 20.

[34] Tamura, R. (2001) Teachers, growth, and convergence. Journal of Political Economy 109(5), 1021-1059.

[35] Uzawa, H. (1965) Optimum technical change in an aggregative model of economic growth. International Economic Review 6, 18-31. 